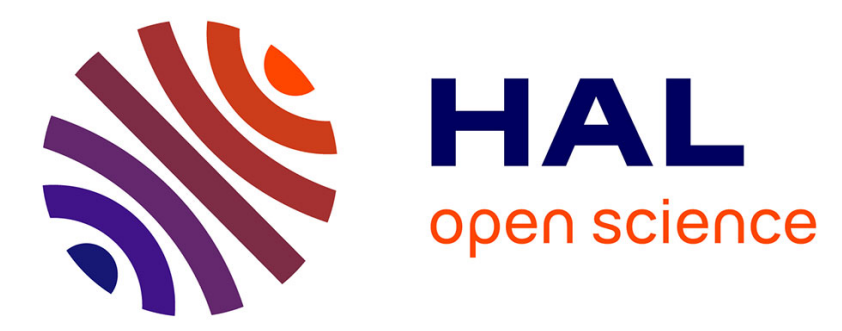

\title{
Les descriptions spatiales "au fond, au sommet, dans le fond, à la base". Entre anaphore associative et anaphore à argument nul \\ Mathilde Salles
}

\section{- To cite this version:}

Mathilde Salles. Les descriptions spatiales "au fond, au sommet, dans le fond, à la base". Entre anaphore associative et anaphore à argument nul. Travaux de Linguistique: Revue Internationale de Linguistique Française, 2011, 2 (63), pp.35-58. 10.3917/tl.063.0035 . hal-02143818

HAL Id: hal-02143818

https://hal-normandie-univ.archives-ouvertes.fr/hal-02143818

Submitted on 12 Jun 2019

HAL is a multi-disciplinary open access archive for the deposit and dissemination of scientific research documents, whether they are published or not. The documents may come from teaching and research institutions in France or abroad, or from public or private research centers.
L'archive ouverte pluridisciplinaire HAL, est destinée au dépôt et à la diffusion de documents scientifiques de niveau recherche, publiés ou non, émanant des établissements d'enseignement et de recherche français ou étrangers, des laboratoires publics ou privés. 


\title{
LES DESCRIPTIONS SPATIALES AU FOND, AU SOMMET, DANS LE FOND, À LA BASE. ENTRE ANAPHORE ASSOCIATIVE ET ANAPHORE À ARGUMENT NUL
}

\author{
Mathilde Salles \\ De Boeck Supérieur | Travaux de linguistique
}

$2011 / 2-n^{\circ} 63$
pages 35 à 58

ISSN 0082-6049

Article disponible en ligne à l'adresse:

http://www.cairn.info/revue-travaux-de-linguistique-2011-2-page-35.htm

Pour citer cet article :

Salles Mathilde, «Les descriptions spatiales au fond, au sommet, dans le fond, à la base. Entre anaphore associative et anaphore à argument nul »,

Travaux de linguistique, 2011/2 n63, p. 35-58. DOI : 10.3917/tl.063.0035

Distribution électronique Cairn.info pour De Boeck Supérieur.

() De Boeck Supérieur. Tous droits réservés pour tous pays.

La reproduction ou représentation de cet article, notamment par photocopie, n'est autorisée que dans les limites des conditions générales d'utilisation du site ou, le cas échéant, des conditions générales de la licence souscrite par votre établissement. Toute autre reproduction ou représentation, en tout ou partie, sous quelque forme et de quelque manière que ce soit, est interdite sauf accord préalable et écrit de l'éditeur, en dehors des cas prévus par la législation en vigueur en France. II est précisé que son stockage dans une base de données est également interdit. 


\title{
LES DESCRIPTIONS SPATIALES AU FOND, AU SOMMET, DANS LE FOND, À LA BASE. ENTRE ANAPHORE ASSOCIATIVE ET ANAPHORE À ARGUMENT NUL
}

\author{
Mathilde SALLES ${ }^{*}$
}

Lorsqu' on prête attention au fonctionnement et à la place de l'anaphore associative dans les textes, les romans en particulier, on voit vite apparaître des relations privilégiées entre ce mode anaphorique et certaines formes de descriptions. Ces relations privilégiées ne sont pas étrangères au mode référentiel propre au défini associatif, comme on le constatera non seulement avec des anaphores associatives décrivant des individus en énumérant des parties de leur corps (type d'anaphore associative pourtant réputé difficile sinon exclu), mais aussi avec des anaphores associatives décrivant des objets et surtout des lieux. Les séquences descriptives spatiales utilisent en effet fréquemment de tels anaphoriques, précédés ou non d'une préposition spatiale. Précédés d'une telle préposition (p.ex. au premier étage, sur le toit), ces anaphoriques associatifs peuvent alors jouer un rôle d'organisateur spatial (Adam, 1993 et 2005), comparable à celui que jouent des adverbes comme devant, dessus et des locutions ou des syntagmes prépositionnels construits avec un "nom de localisation interne » (Borillo, 1988, 1999 ; Aurnague, 1989, 1996) comme au fond, au sommet, sur le dessus.

Se posent alors la question de la place et de l'extension de l'anaphore associative parmi les différents organisateurs spatiaux et la question du statut anaphorique de au fond, au sommet, sur le dessus, formellement très proches des anaphoriques associatifs : s'agit-il d'anaphoriques associatifs ou d'une autre forme d'anaphore ? Sont-ils d'ailleurs tous de même nature ? La double catégorisation précédente, en termes de locutions ou de syntagmes prépositionnels, suggère que non. Pour tenter de répondre à ces différentes questions, un petit rappel de certaines des propriétés qui différencient méronymes et noms de localisation interne s'imposera, rappel qui soulignera

Laboratoire CRISCO (EA 4255 Université de Caen) 
une fois encore ( $c f$. Aurnague, 1996 ; Huyghe, 2005) l'hétérogénéité des noms de localisation interne et la possibilité pour certains d'entre eux de « basculer » vers la méronymie, avec des conséquences sur les possibilités d'emplois anaphoriques (l'alternance le/son, par exemple). On espère ainsi pouvoir mieux comparer les statuts anaphoriques de au fond, sur le dessus, au premier étage, le fond, le dessus, le premier étage, mieux différencier la valeur du défini dans ces différentes expressions et révéler l'existence d'un continuum entre anaphore à argument nul et anaphore associative.

\section{L'anaphore associative et les relations de cohérence descriptives}

Les relations de cohérence descriptives, qu'elles soient essentiellement descriptives comme la relation d'Elaboration d'entité de Prévot, Vieu et Asher (2009) - ou i-élaboration, individual elaboration, chez Fabricius-Hansen et Behrens (2001) - ou partiellement descriptives comme certaines formes de la relation Assertion-indice de Cornish (2009a et b), trouvent une de leurs expressions privilégiées dans l'anaphore associative (désormais AA). L'AA constitue en effet un moyen d'apporter des précisions sur une entité en énumérant les états descriptifs de certaines de ses parties ou composantes. Elle est donc tout naturellement convoquée par Kleiber et Vassiliadou (2007 et 2009) lorsqu'il s'agit d'illustrer la relation d'i-élaboration de FabriciusHansen et Behrens (2001) :

[1] Nous entrâmes dans un village. L'église était située sur une butte. (Kleiber et Vassiliadou, 2007 : 155 ; 2009 : 189)

Dans cet exemple, la deuxième phrase apporte des précisions sur l'entité 'village' introduite dans la première phrase ; l'élaboration ne porte donc pas sur l'événement évoqué par la première phrase, comme c'est le cas lorsqu'on a affaire à une Elaboration « stricte» (l'Elaboration de la SDRT, l'e [ventuality]elaboration de Fabricius-Hansen et Behrens, 2001)1', mais seulement sur l'un de ses actants.

L'AA ne constitue qu'un moyen parmi d'autres de décrire une entité en énumérant ses 'parties' (au sens large du terme ; $c f$. infra), mais elle le fait d'une manière singulière, en détachant les parties qu'elle énumère. On peut, à ce sujet, rappeler les remarques de Kleiber $(1999: 85$; $2001: 242)$ :

« Par opposition à l'individu d'une description complète correspondante [Le $N d u N$ ], celui d'une description $L e N$ en anaphore associative apparaît comme déjà délimité, isolé ou détaché. Il est en quelque sorte aliéné. »

La focalisation qu'opèrent de telles descriptions incomplètes (par opposition aux descriptions complètes $L e N d u N$, mais aussi aux descriptions pos- 
sessives son/sa $\mathrm{N}$ correspondantes) permet des descriptions bien plus spectaculaires, de véritables hypotyposes descriptives comme la suivante ${ }^{2}$ :

[2] Allongée sur le dos, la forme gisait dans le plus complet abandon. Le manteau était ouvert sur le corsage indigo d'une teinte uniforme, avec un étroit col blanc sur le pourtour de la gorge. Le visage de la dormeuse était tourné dans la direction opposée, enfantinement protégé par le bras droit replié. Tout près, une poignée d'anémones était éparpillée dans l'herbe. Une impression infiniment tendre, et en même temps sensuelle émanait de ce corps allongé ; éveillant dans la pensée de Charles quelques vagues échos de son séjour parisien. Une fille, dont le nom à présent lui échappait, qu'il n'avait peut-être jamais su, et qu'il revoyait, endormie ainsi, dans une chambre qui dominait la Seine. (Fowles, Sarah et le lieutenant français, traduction française G. Durand, Points/Seuil, 1981 : 130)

L'hypotypose, qui « peint les choses d'une manière si vive et si énergique, qu'elle les met en quelque sorte sous les yeux, et fait d'un récit ou d'une description, une image, un tableau ou même une scène vivante » (Fontanier, $1977: 390$; je souligne), s'appuie principalement sur les définis associatifs le manteau, le corsage indigo d'une teinte uniforme, la gorge, le bras droit replié. Mais l'effet de gros plan (l'image est de Kleiber, 1999 ; 2001) que produisent ces derniers sur les vetements et parties du corps de Sarah ne contribue pas seulement à peindre énergiquement le «tableau » sensuel qui s'offre à Charles, il contribue aussi à confirmer l'assertion initiale (la forme gisait dans le plus complet abandon), la confirmer en l'explicitant, c'est-à-dire en l'élaborant. On retrouve ici la relation Assertion-Indice de Cornish, (2009a et b), qui comprend une partie élaborante, « sémantique » (concernant les contenus propositionnels des unités reliées) et une partie argumentative, «pragmatique $»^{3}$ (concernant les relations entre les actes illocutoires associés à ces unités) :

\section{Assertion-Indice :}

1. Inférer $P$ de l'assertion de $S^{0}$ ainsi que de $S^{1}$, où $S^{1}$ ajoute d'autres détails à $P$ et $\mathrm{e}^{1} \subseteq \mathrm{e}^{0}\left[\mathrm{e}^{1}\right.$ et $\mathrm{e}^{0}$ sont les événements principaux évoqués par $\mathrm{S}^{1}$ et $\mathrm{S}^{0}$ ] (= la relation Elaboration)

2. Interpréter $\mathrm{S}^{1}$ comme rendant plus convaincante l'hypothèse du locuteur qui correspond à l'assertion de $S^{0}$. (Cornish 2009b : 169)

La première partie de la définition de Cornish correspond ainsi à la définition de la relation Elaboration, fondamentalement " sémantique » (cf. Cornish, 2009b : 172, note 19), c'est-à-dire se fondant en priorité sur le contenu sémantique des unités. En l'occurrence, il s'agit de spécifier le contenu sémantique d'une première unité en ajoutant un ou des détails sup- 
plémentaires sur la situation décrite. La deuxième partie de sa définition souligne la valeur argumentative de la deuxième unité, destinée à accroître la conviction de l'interlocuteur ou du lecteur.

La description de [2] est un exemple de cette relation de cohérence : une série de prédicats statifs é-élaborent un prédicat statif initial, en décrivant des 'parties' d'un actant (ce qui relève en outre d'une Elaboration d'entité ou i-élaboration), et s'interprètent comme autant de preuves particulières d'une assertion plus générale : l'état d'abandon de Sarah est ainsi confirmé par l'état de ses vêtements et la position de son corps, entre autres ${ }^{4}$. Dans cet exemple, l'AA permet une forme d'imbrication de l'é-élaboration et de l' $i$ élaboration, dans la mesure où elle permet de préciser et ainsi de certifier l'état d'une entité par l'état de telle(s) ou telle(s) de ses parties. Cette forme particulière de la relation Assertion-Indice suppose une continuité aspectuelle (l'Assertion et ses Indices sont des états) et trouve une expression privilégiée avec l'AA, que celle-ci soit utilisée pour la description d'humains (ex. [2] et [3]), de lieux (ex. [4] et [5]) ou encore d'entités collectives comme la caravane de [6] :

[3] Hugues recommençait chaque soir le même itinéraire, suivant la ligne des quais, d'une démarche indécise, un peu voûté déjà, quoiqu'il eût seulement quarante ans. Mais le veuvage avait été pour lui un automne précoce. Les tempes étaient dégarnies, les cheveux pleins de cendre grise. (G. Rodenbach, Bruges-la-morte, Bruxelles, Babel, $1989: 25)$

[4] M. Lebigre tenait un fort bel établissement, d'un luxe tout moderne. Placé à l'encoignure droite de la rue Rambuteau, flanqué de quatre petits pins de Norvège dans des caisses peintes en vert, il faisait un digne pendant à la grande charcuterie des Quenu-Gradelle. Les glaces claires laissaient voir la salle, ornée de guirlandes de feuillages, de pampres et de grappes, sur un fond vert tendre. Le dallage était noir et blanc, à grands carreaux. Au fond, le trou béant de la cave s'ouvrait sous l'escalier tournant, à draperie rouge, qui menait au billard du premier étage. Mais le comptoir surtout, à droite, était très riche, avec son large reflet d'argent poli. (Zola, Le ventre de Paris, Folio/ Gallimard, $2002:$ 168)

[5] Mais la construction de grands entrepôts avait bloqué cette perspective, et il paraissait évident que ces demeures avaient perdu toute confiance en leur élégance naturelle. La peinture des charpentes s'était écaillée, les toitures de tuile, les panneaux de porte se fissuraient. (Fowles, Sarah et le lieutenant français, traduction française G. Durand, Points/Seuil, 1981 : 398)

[6] Une caravane, qui a traversé à gué, nous arrive dans un état invraisemblable ; les chameaux, enduits jusqu' aux yeux de boue gluante, sont devenus des monstres informes et squameux ; quant aux mules 
qui les accompagnaient, elles se sont, paraît-il, noyées dans la vase. (Loti, Vers Ispahan, Frantext)

Dans chacun de ces exemples, l'assertion initiale précise l'état d'une entité que la ou les propositions qui suivent prouvent par l'exemple : ainsi, le vieillissement précoce du personnage, effet de son veuvage, est confirmé par les propriétés de certaines de ses parties du corps en [3]. Le luxe de l'établissement de M. Lebigre en [4] et la dégradation des demeures en [5] sont de la même façon confirmés par les propriétés de différentes 'parties' 'parties' au sens large, puisqu'on trouve à la fois des AA méronymiques (le premier étage) et des AA locatives (le comptoir), suivant la classification de Kleiber $(2001)^{5}$. Enfin, l'état invraisemblable dans lequel se trouve la caravane est prouvé par l'état de certains de ses membres en [6]. ${ }^{6}$

La relation Assertion-Indice, lorsqu'elle relie des états descriptifs, ne manifeste pas la tendance, remarquée par Prévot, Vieu et Asher (2009 : 214) à propos de la relation d'Elaboration d'entité, à présenter plutôt des propriétés permanentes que des propriétés transitoires. Tout dépend en fait du type de propriété asserté dans la proposition initiale : un état transitoire d'une entité globale sera prouvé par un ou des états transitoires de certaines de ses parties, alors qu'un état permanent le sera par un ou des états permanents de parties. La seule régularité qu'on observe est liée à la nature visuelle de la preuve : même si le prédicat statif initial n'est pas explicitement visuel, les prédicats statifs suivants, qui servent à justifier le premier, sont des descriptions visuelles. Or, plus que n'importe quelle autre manière de présenter des référents associatifs, l'AA, par l'effet de détachement ${ }^{7}$ qu'elle implique, contribue à promouvoir la partie au statut d"évidence', de preuve visible. Cela explique son affinité particulière avec cette forme de la relation Assertion-Indice, avec l'hypotypose et, plus généralement, avec les séquences descriptives.

\section{AA et structuration textuelle de l'espace}

\subsection{Les listes descriptives spatiales}

Comme l'a souligné Adam (1993 et 2005), les descriptions se caractérisent par une présence importante d'organisateurs textuels, spatiaux (à gauche / à droite, devant / derrière, dessus / dessous, en haut / en bas, au nord / au sud...), temporels et/ou énumératifs (d'abord, ensuite, (et) puis, (et) enfin, alors, après ). Dans les listes descriptives spatiales, ces organisateurs textuels sont fréquemment des AA méronymiques ou locatives précédées d'une préposition spatiale, comme sous l'escalier tournant en [4], au second étage, sur la plate-forme supérieure, dans la célèbre description de la pièce montée de Mme Bovary donnée en [7], ou alors des locutions formellement très proches construites avec un «nom de localisation interne $»^{8}$ 
(Borillo, 1988 et 1999, Aurnague, 1989 et 1996), comme au fond en [4], à la base, au sommet, en [7] :

[7] On avait été chercher un pâtissier à Yvetot, pour les tourtes et les nougats. Comme il débutait dans le pays, il avait soigné les choses ; et il apporta, lui-même, au dessert, une pièce montée qui fit pousser des cris. À la base, d'abord, c'était un carré de carton bleu figurant un temple avec portiques, colonnades et statuettes de stuc tout autour, dans des niches constellées d'étoiles en papier doré ; puis se tenait au second étage un donjon en gâteau de Savoie, entouré de menues fortifications en angélique, amandes, raisins secs, quartiers d'oranges ; et enfin, sur la plate-forme supérieure, qui était une prairie verte où il y avait des rochers avec des lacs de confitures et des bateaux en écales de noisettes, on voyait un petit Amour, se balançant à une escarpolette de chocolat, dont les deux poteaux étaient terminés par deux boutons de rose naturels, en guise de boules, au sommet ${ }^{9}$. (Flaubert, Madame Bovary, Folio/Gallimard, 1985 : 53 ; cité par Adam $1993: 99$ et $2005: 118$ )

Le statut de locution (adverbiale ou, selon Borillo, prépositionnelle ; voir notamment Borillo 1992 : 185) est toutefois contesté par le critère que retient Adler (2006 et 2008) pour distinguer syntagmes libres et suites lexicalisées, c'est-à-dire l'équivalence sémantique entre [PREP $L E$ NOM $D E$ ] et $[L E$ NOM $D E]$. Une telle équivalence (par exemple, entre au fond de l'établissement et le fond de l'établissement, entre à la base / au sommet de la pièce montée et la base / le sommet de la pièce montée) permet de conclure, selon Adler, que au fond de, au sommet de, etc. sont des syntagmes libres et non des locutions et aussi d'analyser au fond, à la base, au sommet, comme des AA. On aimerait cependant pouvoir convoquer d'autres critères que la simple équivalence sémantique évoquée par Adler pour distinguer syntagmes libres et expressions lexicalisées. Un relecteur propose l'adjonction d'une modification syntaxique interne à la locution ou au syntagme (p.ex. *au fond sombre de l'établissement), adjonction normalement exclue dans les locutions. Mais cette adjonction se révèle souvent difficile avec le défini - indépendamment de tout figement - à cause des effets contrastifs inappropriés qu'elle peut produire (le fond sombre, opposé à un autre fond ; sur ce point, voir Theissen, 2001a et b). Une solution pourrait être d'ajouter un syntagme apposé : *au fond, sombre /le fond, sombre, *au sommet, enneigé / le sommet, enneigé, avec ici des différences qui rendent bien contestable l'équivalence sémantique évoquée par Adler ; on y reviendra.

Les listes narratives seront, quant à elles, essentiellement structurées par des organisateurs temporels, comme les adverbes d'abord, ensuite, qu'on trouve en [8], les locutions ou $\mathrm{AA}^{10}$ au début, à la fin, etc. : 
[8] Terre, elle, d'abord enfanta un être égal à elle-même, capable de la couvrir tout entière, Ciel Étoilé, qui devait offrir aux dieux bienheureux une assise sûre à jamais. [...] Mais ensuite, des embrassements du Ciel, elle enfanta Océan aux tourbillons profonds [...] (début de la Théogonie d'Hésiode, traduction française P. Mazan ; cité par Eco, 2009 : 19) :

C'est exclusivement aux organisateurs spatiaux qu'on s'intéressera ici, et, parmi ces derniers, à ceux qui utilisent une AA méronymique ou locative et à ceux qui, tels au fond, à la base, au sommet, sont, sinon assimilables à une AA, formellement proches de cette anaphore. Pour tenter de démêler les ressemblances et différences entre ces deux types d'organisateurs spatiaux, commençons par un petit rappel de la littérature consacrée aux noms de localisation interne (désormais Nli) et à leurs ressemblances-différences avec les méronymes.

\subsection{Méronymes et noms de localisation interne (Nli)}

Les jugements sur la capacité ou non des Nli et des méronymes à entrer dans telle ou telle construction ne font pas toujours l'unanimité. Borillo ellemême exclut plus (Borillo, 1992) ou moins (Borillo, 1998 et 1999) la possibilité de pronominaliser le complément du Nli par un possessif et accepte plus (1999) ou moins (1988 et 1998) ce même type de pronominalisation lorsqu'il s'agit du complément d'un méronyme ${ }^{11}$. Ainsi, on peut effectivement opposer la possibilité de pronominaliser le complément des méronymes en [9] à la difficulté sinon l'impossibilité d'opérer une telle pronominalisation avec des Nli en [10] :

[9] a. le toit de la maison $\rightarrow$ son toit

b. la porte du magasin $\rightarrow$ sa porte

[10] a. le haut / bas de la maison $\rightarrow$ ?son haut / bas

b. le fond du magasin $\rightarrow$ ?son fond

Mais d'autres Nli, comme extrémité, centre, sommet, selon Borillo (1998), ou intérieur, pourtour, rebord, sommet, selon Borillo (1999) ${ }^{12}$, accepteraient mieux le possessif que les trois Nli précédents (haut, bas, fond)... Décontextualisé, son pourtour ne paraît pourtant guère plus acceptable que son avant (marqué «*? » par Borillo, 1988), peut-être même un peu moins. Et que dire de son septentrion? Voici la réponse de Jean Echenoz:

[11] A l'abandon, le château d'eau s'écaillait ; une colonne de mousse rase rongeait en triangle son septentrion. (Echenoz, L'équipée malaise, Minuit «Double», $2006: 234$ )

Il semblerait finalement qu'on puisse uniquement souligner une régularité (ce que fait d'ailleurs Borillo, 1998) : le défini - autrement dit l'AA ${ }^{13}$ 
ou ce qui y ressemble fort - est le mode anaphorique privilégié des Nli, l'anaphore possessive restant marginale. Quant aux difficultés à estimer l'acceptabilité de telle ou telle construction avec des Nli et aux désaccords qui en découlent, ils paraissent liés au caractère hétérogène de la catégorie et à la prédominance des emplois prépositionnels avec article défini (avec en plus des cas de figement assez nets).

\subsubsection{L'hétérogénéité de la catégorie des $\mathrm{Nli}$}

L'hétérogénéité de la catégorie a conduit Aurnague (1996) à poser un continuum entre Nli proprement dits et noms de composants. Par exemple, arête, un des Nli de Borillo (1988 : 18), serait plus proche des noms de composants que angle, coin, parce qu'il refuse la préposition $\grave{a}^{14}$ :

[12] Il y a un écriteau à l'angle / au coin / *à l'arête du mur. (Aurnague 1996 : 181)

Mais ce n'est apparemment pas cette hétérogénéité-là qui permet d'expliquer pourquoi un Nli comme sommet (Nli au sens strict selon Aurnague) accepte assez bien (selon moi) certaines constructions que sont censés refuser les Nli et que sont censés accepter les méronymes. Par exemple, la pronominalisation du Nli par celui (cf. Borillo, 1992, Aurnague, 1996), si elle se révèle effectivement difficile pour le Nli haut, ne l'est pas toujours pour sommet :

[13] a. Le haut du Mt Blanc est couvert de neige. ?Celui du Mt Maudit l'est aussi.

b. Le sommet du Mt Blanc est couvert de neige. Celui du Mt Maudit l'est aussi.

Et, contrairement à Aurnague (1996), la pronominalisation par le démonstratif me semble bien plus acceptable avec sommet qu'avec haut dans l'exemple suivant (présenté ici avec le jugement d'acceptabilité de son auteur) ${ }^{15}$ :

[14] Le corbeau est sur le sommet / haut de la tour. ??La corneille est sur celui du clocher.

(Aurnague $1996: 178$ )

Mais elle ne l'est plus dans [15], qui, on le verra, présente une construction prépositionnelle (au sommet de) plus figée que la précédente ${ }^{16}$ :

[15] Des corbeaux nichent au sommet de la tour et des pigeons ??à celui du clocher.

Le statut plus ou moins figé des séquences préposition $+N l i+d e$ est donc un autre élément perturbateur. 
Quelle différence, toutefois, entre haut et sommet, si l'on veut bien admettre que ces deux Nli ne se comportent pas de la même manière en [13] et [14] ? La réponse est peut-être liée à une différence de " généralité » (cf. Aurnague, 1996) : «Le caractère général des Nli » et, ajoute Aurnague (1996 : 176), « la non identification fonctionnelle et géométrique qui en découle » expliquent pourquoi ils refusent les emplois autonomes, i.e. les emplois sans référence (explicite ou anaphorique) à un tout. Aurnague (1996) et Huyghe (2005) excluent ainsi des emplois comme c'est un fond / un haut; ce fond / haut est sale; qu'est-ce qu'un fond / haut $?{ }^{17}$ Mais peut-on exclure de la même manière ce sommet reste enneigé toute l'année; ce sommet est le plus haut de la ville; qu'est-ce qu'un sommet / un rebord? Ces emplois autonomes ne paraissent pas impossibles, avec des Nli un peu moins généraux, capables d'identifier les positions d'objets particuliers sans qu'il soit nécessaire de mentionner ces objets. Contrairement à ce haut, ce sommet pourra référer à la partie la plus haute d'une montagne ou d'un immeuble. Des informations contextuelles appropriées (par exemple enneigé) suffisent à identifier le 'bon' sommet, pas le 'bon' haut : ?ce haut est enneigé est bien moins acceptable que ce sommet est enneigé. Mais ce sommet ne pourra vraisemblablement pas référer à la partie supérieure d'une armoire ou d'une boîte : ?ce sommet est poussiéreux versus le sommet de l'armoire / de la boîte est poussiéreux. Les emplois autonomes de sommet seraient donc limités à certains sommets (plus caractéristiques, plus spécifiques ?).

La généralité n'explique toutefois pas tout, car un Nli plus spécialisé ${ }^{18}$ comme lisière, par exemple, n'accepte pas mieux ou moins bien ces emplois autonomes qu'un Nli plus général comme bordure. Qu'est-ce qu'une lisière / une bordure ? semblent également possibles. Même chose pour c'est une lisière / une bordure, cette lisière / bordure s'effiloche, à condition cette fois de parler de la lisière / bordure d'une pièce de tissu, d'une voile (pour bordure) ou d'un vêtement, et non de la lisière / bordure d'un bois ou d'un champ. La différence semble ici liée à la matérialité des zones désignées (cf. Huyghe, 2005 : 199-201, sur ce point) : certaines ne sont que zones (milieu, limite, ou encore bordure et lisière comme Nli de bois ou champ), c'est-àdire qu'elles se caractérisent par une référence exclusivement spatiale, sans référence matérielle ; d'autres sont à la fois zones et parties matérielles, combinant ainsi référence spatiale et référence matérielle (avant, dessus, sommet, ou encore bordure comme Nli de voile, bordure et lisière comme Nli de tissu).

L'hétérogénéité sémantico-référentielle des Nli serait ainsi le premier facteur expliquant la difficulté à s'accorder sur le comportement syntaxique des Nli. Généralité sémantique et absence de référence matérielle bloqueraient la plupart des constructions évoquées, les Nli qui présentent la seconde caractéristique, c'est-à-dire ceux qui désignent de pures zones, 
semblant d'ailleurs être les moins autonomes, les plus « dépendants » au sens où l'entend Husserl (1962) lorsqu'il oppose les contenus dépendants, qui refusent toute représentation indépendante, et les contenus indépendants. Alors que même les parties les plus inaliénables, telles les parties du corps, sont des contenus indépendants, représentables séparément, les propriétés sont, elles, des contenus dépendants, qu'on ne peut se représenter séparément d'un « substrat » : si, écrit Husserl (1962: 24), « on peut assurément se représenter une tête séparée de l'homme auquel elle appartient $^{19}$, on ne peut se représenter de cette manière une couleur, une forme, etc., elles ont besoin d'un substrat, dans lequel on les remarque sans doute exclusivement mais dont elles ne pourront être séparées ». La représentation indépendante de zones dépourvues de matérialité comme la lisière ou le milieu d'une forêt est, elle aussi, exclue. Le défaut de congruence ontologique (Kleiber, 1999 et 2001) entre la zone et le tout est comparable à celui qu'on peut observer entre des propriétés comme la longueur, la hauteur, la couleur, le poids et leur entité de rattachement. Dans les deux cas, les éléments ne sont pas du même type ontologique que le tout : par exemple, la lisière ou le milieu d'une forêt sont, contrairement à la forêt, dépourvus de matérialité, et la couleur d'une voiture n'a, contrairement à la voiture, ni forme ni matière propres. Mais cette absence d'autonomie et ce défaut de congruence ontologique n'ont pas tout à fait les mêmes incidences linguistiques selon qu'on a affaire à un nom de zone ou un nom de propriété. Avec les noms de propriétés, l'absence d'autonomie se traduit tout naturellement par la difficulté d'utiliser des structures impliquant l'autonomie, des structures aliénantes, ne mentionnant pas l'entité de rattachement de la propriété, comme l'AA [16 $]^{20}$. Alors que les structures qui mentionnent, sous une forme ou une autre (déterminant possessif, pronom en, syntagme prépositionnel), l'entité de rattachement, c'est-à-dire les structures qui « conserve[nt] un indicateur anaphorique de la dépendance ontologique » (Kleiber, $2001: 254)$ ne posent plus de problèmes [17] :

[16] Max a acheté une voiture. ?La couleur est étonnante.

[17] Max a acheté une voiture. Sa couleur / La couleur en / La couleur de cette voiture est étonnante.

Avec nos Nli de zones, AA et possessif semblent également rares ou, si l'on ose encore se prononcer sur des exemples forgés, également difficiles. Ainsi un Nli comme milieu n'est guère meilleur avec son qu'avec le en [18] :

[18] Nous entrâmes dans un bois. ?Le milieu / ?Son milieu semblait inaccessible.

Les rares occurrences de son + Nli milieu et le + Nli milieu relevées dans une petite sélection de textes (les soixante-quatre romans publiés entre 1987 et 
2007 compris dans la base Frantext non catégorisée) sont toutes prépositionnelles (prépositions spatiales en, avec son, et par, avec son ou le) : Le grand pont flottant était ouvert en son milieu (Rambaud, La Bataille), le terreplein qui scindait le boulevard par son milieu (Jonquet, Les Orpailleurs), Un lavabo fendu en deux par le milieu (Jonquet, Les Orpailleurs). Et une rapide recherche des occurrences de lisière dans la même sélection de textes confirme que ni l'AA ni le possessif ne font partie des emplois réguliers de lisière. Sur les 53 occurrences relevées, trois seulement peuvent être considérées comme des anaphores - associatives [19]-[20] ou possessive [21] :

[19] Il ne sortait de sa cabane que pour aller déambuler vers la forêt, mais n'y pénétrait jamais. Il s'arrêtait au seuil des arbres et longeait la lisière. (Germain, Jours de colère, Frantext)

[20] Surpris qu'il fermât la porte derrière lui, je l'interrogeai du regard ; je me trouvais dans le rectangle de lumière, et lui, en retrait, à la lisière, haletant [...] (Bianciotti, Le pas si lent de l'amour, Frantext)

[21] J'étais là, prêt à un certain danger, à sa lisière, en voyeur, en être passif des expériences. (Guibert, L'incognito, Frantext)

Trois, cela semble bien peu comparé à l'importance des emplois avec complément prépositionnel en de. Ces derniers, la lisière de ou lisière de sans déterminant après la préposition en, représentent en effet 46 des 53 occurrences $^{21}$.

Qu'en est-il des Nli ontologiquement congruents ? Rien ne s'oppose a priori à un détachement par le défini associatif. Le défini serait même, on l'a noté à la suite de Borillo, mieux approprié à la reprise anaphorique que le possessif :

[22] a. Cette voiture est vieille, les ceintures ne sont qu'*à son avant / à l'avant.

b. Prenez cette bouteille, il y a du vin dans *son fond / le fond. (exemples de Borillo, 1998 : 75 ; les jugements d'acceptabilité sont les siens)

Et Borillo (1998: 75) précise que si l'« on a peut-être encore le choix pour des noms comme extrémité, centre, sommet [...], l'article devient pratiquement obligatoire avec avant, bas, haut, fond, etc. ».22 Mais tous les exemples de Borillo (1998) sont prépositionnels (à l'avant, dans le fond....). En dehors de ces constructions, observe-t-on le même rejet du possessif au profit du défini ? Dans une séquence comme [23], avec le Nli avant, défini et possessif semblent également possibles ${ }^{23}$ :

[23] Max a acheté une voiture. L'avant / Son avant ressemble à l'avant / celui d'une Maserati. 
Et une recherche du Nli fond dans Frantext confirme que le possessif, aussi rare qu'il soit, n'est pas exclu dans des constructions non prépositionnelles. La recherche a été effectuée sur la même sélection que précédemment (64 romans, 1987-2007). Dans cette sélection, on relève 1650 occurrences de fond, parmi lesquelles 845 au fond, 257 le fond et seulement 3 son fond, dont 1 son fond de teint, ce qui réduit les combinaisons possessif + Nli fond à 2 seulement :

[24] [...] en rentrant les bannes d'osier, la Comtesse vit que le nuage s'élevait et s'étalait ; il s'assombrissait, son fond était très noir mais au sommet il semblait presque vert. (Chandernagor, L'Enfant des lumières, Frantext)

[25] Un jour, il parla d'un petit lac, en pleine taïga, gelé onze mois sur douze. Par volonté de leur chef de camp, son fond s'était transformé en cimetière : c'était plus simple que de creuser le permafrost. (Makine, Le Testament français, Frantext)

Les emplois non prépositionnels du possessif sont rares, mais les emplois non prépositionnels du défini anaphorique ne sont pas bien nombreux non plus. Sur les 257 occurrences de le fond relevées dans Frantext, seules 10 occurrences non prépositionnelles peuvent être traitées comme des Nli anaphoriques, le plus souvent associatifs [26]-[27], occasionnellement coréférentiels [28] :

[26] [...] nous avons lancé un défi : plonger dans l'Allier, tête la première, d'une courte butte de graviers et de boue sur les bords. L'eau était opaque totalement et le fond invisible. (Schreiber, Un silence d'environ une demi-heure, Frantext)

[27] Ce papier était déjà ancien, fané, assez sali et même écorché par endroits. Le rouge et l'orangé des roses peintes avaient bruni, et le fond, qui avait dû être d'un léger jaune paille s'était encrassé. (Germain, La Pleurante des rues de Prague, Frantext ; c'est cette valeur de fond, synonyme d'arrière-plan et rapporté à un papier peint, un tableau ou un paysage, qu'on trouve dans la plupart des exemples anaphoriques non prépositionnels de le fond)

[28] Je glisse le portrait entre les pages du cahier et je range le cahier dans le fond du placard. Le fond est jaune clair, bouton d'or. (Belloc, Néons, Frantext)

Une onzième occurrence non prépositionnelle de le fond mérite d'être évoquée. Il s'agit d'un emploi de le fond dans une construction attributive du verbe avoir, construction caractéristique de la méronymie stricte et, comme l'a souligné Kleiber (2001), inappropriée aux relations locatives (p.ex. village / église, cuisine / réfrigérateur) : 
[29] Pour sortir j' avais un problème délicat à régler... mon froc glorieux, celui rescapé de la bataille de Colmar. Il avait le fond en lambeaux. (Boudard, Mourir d'enfance, Frantext)

Dans cet exemple, fond n'est plus un Nli mais un méronyme, et c'est d'ailleurs pour cette raison qu'il accepte les « emplois typifiants de la forme un Nli de $N$ » évoqués par Huyghe (2005 : 193) : un fond de pantalon, un fond d'artichaut, un bas de survêtement (exemples repris à Borillo, 1999). Dans ces trois exemples, fond et bas sont des méronymes, comme le souligne fort justement Borillo (1999 : 65) ${ }^{24}$.

La construction attributive du verbe avoir pourrait se révéler un bon critère pour distinguer Nli et méronymes, meilleur peut-être que l'utilisation du possessif ou de la pronominalisation par celui. Ce qui apparaît lorsqu'on tente d'évaluer des exemples forgés - exercice périlleux, on l'a constaté à plusieurs reprises - ce sont différentes positions de tel ou de tel nom ou de tel ou tel emploi sur un continuum comparable à celui d'Aurnague (1996), allant des Nli strictement topologiques aux méronymes, en passant par des Nli plus méronymiques. Par exemple, [30a] et [31a] présentent des valeurs plus méronymiques de coins et bordure que [30b] et [31b], des valeurs plus méronymiques qui, non seulement autorisent la construction attributive du verbe avoir, mais semblent aussi autoriser les emplois typifiants évoqués plus haut (un coin de table, une bordure de voile) :

[30] a. Cette table a les coins abîmés.

b. ? Cette pièce a les coins / le fond moisi(s).

[31] a. Cette voile a la bordure qui s'effiloche.

b. ?? Ce bois a la bordure / la lisière située tout près de l'autoroute.

\subsubsection{La prédominance des emplois prépositionnels des Nli}

La difficulté qu'on peut éprouver à estimer certaines des séquences de Borillo, Aurnague ou Huyghe, et ainsi à évaluer la pertinence des critères est parfois liée au caractère peu naturel de ces séquences. La fonction des Nli, inhérente à leur valeur sémantique, est de localiser. Les emplois de Nli sont donc très généralement des emplois localisants : les Nli sont le plus souvent compléments d'une préposition locative, quelquefois compléments d'un verbe de mouvement ${ }^{25}$. Dans les autres emplois, la dimension locative des Nli s'efface plus ou moins au profit d'une valeur méronymique. La position de sujet, par exemple, contribue à présenter fond davantage comme un objet, un composant que comme un lieu dans les exemples [26] à [28]. Mais ces exemples restent rares, comme le révèle une rapide comparaison du nombre d'occurrences relevées dans Frantext. Parmi les emplois anaphoriques de le fond, on relève seulement 8 occurrences de le fond non précédées d'une préposition et non compléments d'un verbe de mouvement 
pour 27 occurrences prépositionnelles (par ordre de fréquence : dans le fond, vers le fond, sur le fond $)^{26}$ et 2 occurrences compléments d'un verbe de mouvement (rejoindre, atteindre). En outre, 5 de ces 8 occurrences désignent l'arrière-plan d'un tableau, d'un paysage ou d'un papier peint ( $c f$. exemple [27]), type d'emploi de fond qui, semble-t-il, se prête plus facilement à la «méronymisation».

Même rareté pour le sommet, dont on ne relève que 3 emplois anaphoriques non précédés d'une préposition et non compléments d'un verbe de mouvement sur 39 occurrences de la forme le sommet ${ }^{27}$. Ces emplois seront encore plus rares, sinon exclus, pour des Nli de zone (p.ex. lisière, bordure / bois, champ). Leur absence de partie matérielle ne les prédispose pas à être présentés autrement que comme des lieux. Ici se dessine une différence importante entre les emplois anaphoriques qui ne sont compléments ni d'une préposition ni d'un verbe de mouvement et les autres emplois anaphoriques, compléments d'une préposition ou d'un verbe de mouvement. Seuls les premiers gomment la valeur locative des Nli. Les autres la soulignent soit en faisant du Nli le complément d'un élément spatial (préposition spatiale ou verbe de mouvement : au sommet, atteindre le sommet), soit en utilisant le Nli lui-même comme un complément du nom localisant (le mur $d u$ fond, les chambres du bas). Ces derniers emplois sont assez fréquents. Par exemple, notre sélection de romans, qui présentait seulement 8 le fond anaphoriques, propose $39 \mathrm{du}$ fond associatifs textuels ou situationnels ${ }^{28}$. Si l'un est complément d'un verbe de mouvement (venir), les autres sont tous compléments du nom ou du pronom (un cas avec celles $d u$ fond, pour les pièces du fond). Il est intéressant de noter que ces noms, dont $d u$ fond est le complément, sont généralement (33 des 38 occurrences) des méronymes ou des noms de $«$ localisés fonctionnels ${ }^{29} \mathrm{du}$ nom auquel se rattache luimême le Nli fond. Par exemple, mur, paroi, méronymes de pièce, chambre, dans le mur du fond, la paroi du fond [de la pièce / de la chambre], ou encore banquette, tables, noms de localisés fonctionnels de bar, restaurant dans la banquette du fond, les tables du fond [du bar / du restaurant]. Du fond sert donc principalement à identifier une 'partie' ${ }^{30} \mathrm{~d}$ ' un tout en la localisant dans ce dernier.

Certains Nli seront donc plus ou moins localisateurs, selon la construction dans laquelle ils entreront : les constructions non localisantes auront tendance à activer une dimension plus méronymique de ces noms, la plupart des constructions prépositionnelles souligneront au contraire leur valeur de localisation interne ${ }^{31}$. De façon plus précise encore, en considérant le figement plus accompli des formations en $\grave{a}$ ( $c f$. section suivante), on peut évoquer les « trois niveaux d'actualisation du sens des noms de position » identifiés par Huyghe (2005 : 203) :

«(i) Le nom de position renvoie à une partie matérielle : Le bord de la table est en bois / fendu 
(ii) Le nom de position s'emploie comme localisateur (notamment avec dans et sur) : Le verre est sur le bord de la table [...]

(iii) Le nom de position donne une instruction de configuration dans une locution prépositionnelle (avec à / en) : Le verre est au bord de la table. »

Inversement, certains noms habituellement traités comme des méronymes ou des localisés fonctionnels acquerront plutôt une valeur de localisateur lorsqu'ils entreront dans certaines constructions prépositionnelles (p.ex. au second étage dans la description de la pièce montée donnée en [7]).

\subsubsection{Un continuum anaphorique}

Cette manière de présenter le référent - comme un lieu ou comme une partie - a des incidences sur le mode d'aliénation opéré par le défini anaphorique. «Aliénation » n'est d'ailleurs plus un terme très approprié lorsqu'on a affaire à des lieux. La « focalisation » n'est plus du même ordre et, si l'on veut filer la métaphore de Kleiber (2001), il ne s'agit finalement plus de faire des gros plans, de changer la focale, mais de déplacer la caméra. D'opérer un changement topologique, une forme de travelling. Peut-on alors encore parler d'AA pour des anaphores comme au fond, dans le fond, au sommet, qui ne présentent pas, à proprement parler, leur référent comme aliéné par rapport au référent de l'antécédent ? Ou ne devrait-on pas les traiter comme des « anaphores argumentales nulles, se référant à une séquence précédente dans le contexte », traitement qu'Adler (2008:68) réserve aux séquences lexicalisées formant des «blocs prépositionnels »? L'argument d'Adler (2006 et 2008) pour traiter au fond, dans le fond, au sommet, etc., comme des AA, anaphores reposant sur une relation préétablie ( $c f$. Kleiber, 2001), et non comme des anaphores argumentales nulles, dans lesquelles la relation entre le complément (effacé) et le bloc prépositionnel se fait syntaxiquement et non pas précontextuellement, repose sur l'équivalence sémantique entre [PREP $L E$ NOM $D E]$ et [ $L E$ NOM $D E]$. Avec une telle équivalence, l'anaphore reste nominale : il s'agit bien d'un SN anaphorique défini (le fond, le sommet) - qui, conformément aux propriétés de l'AA, introduit un nouveau référent par l'intermédiaire d'une entité mentionnée auparavant dans le texte - et non de l'ellipse de l'argument d'un bloc prépositionnel (au fond (de $\emptyset)$, au sommet (de $\emptyset)$ ), comme dans le cas de l'anaphore à argument nul. Mais peut-on vraiment parler d'équivalence entre au fond de la cour et le fond de la cour, entre au fond et le fond des exemples [26]-[28] ? Contrairement à ce qu'affirme Adler (2006 : 260), il n'est pas certain que sur le dessus de (qu'elle oppose ici à au-dessus de) « continue à indiquer une partie intégrante de l'objet dénoté par $\mathrm{X}$, à l'égal du $\mathrm{SN}$ le dessus de $X »$. Les différences de pronominalisation semblent au contraire refléter une différence entre le dessus de, plus méronymique, et sur le dessus de, moins 
méronymique. En effet, si le dessus de autorise la pronominalisation de son complément par un possessif [34], une telle pronominalisation semble plus difficile avec sur le dessus de [33b] ; en tout cas, elle se révèle plus rare ${ }^{32}$ :

[33] a. Tu as vu le petit meuble que j'ai à côté de la porte ? Pour que ton mari ne rate pas le message que tu lui as écrit, tu peux le coller sur le dessus. (exemple proposé par Adler $2006: 262$ )

b. [...] ?? tu peux le coller sur son dessus.

[34] [...] il [=le petit buffet] contient aussi le sucre, le café, les confitures en voie d'extinction (ce n'est pas l'Armoire aux Confitures, qui est, elle, au grenier) ; son dessus est encombré d'une véritable armée de médicaments [...] (Roubaud, Le grand incendie de Londres : récit, avec incises et bifurcations, Frantext)

La seule occurrence de sur son dessus relevée dans l'intégralité de la base Frantext non catégorisée (4016 textes) comprend une détermination nominale (de marbre blanc veiné) qui redonne justement à dessus une valeur plus méronymique, plus proche de [34] que de [33] :

[35] [...] un grand chiffonnier à sept tiroirs, d'époque Louis XVI, en acajou mouluré et filets en bois de couleur ; sur son dessus de marbre blanc veiné sont posés deux chandeliers à dix branches, un tailloir d'argent [...] (Perec, La Vie mode d'emploi, Frantext)

Avec certaines formations en à, le possessif se révèle plus difficile encore, signe que le figement est plus accompli ${ }^{33}$ :

[36] M. Lebigre tenait un fort bel établissement, d'un luxe tout moderne. *A son fond, le trou béant de la cave s'ouvrait sous l'escalier tournant, à draperie rouge, qui menait au billard du premier étage.

[37] Tu as vu le petit meuble que j'ai à côté de la porte ? A l'intérieur / *A son intérieur, tu trouveras le double des clefs de l'appartement.

D'autres formations en $\grave{a}+$ Nli connaissent des emplois possessifs ; c'est le cas de à son sommet dont on trouve plusieurs occurrences dans notre petite sélection de romans :

[38] La tour carrée de l'église avait été éclatée à son sommet par un obus. (Rambaud, La Bataille, Frantext)

[39] [à propos d'un meuble] les ronds de bière calant son pied avant droit, les cartons à chapeaux que l'on imagine si bien même s'ils n'y sont plus, s'entassant de guingois à son sommet [...] (Benoziglio, La Voix des mauvais jours et des chagrins rentrés, Frantext)

Sans doute est-ce là l'indice que les formations en $\grave{a}+l e$ Nli présentent des différences de degré de figement. Mais, toutes ces formations en $\grave{a}+l e$ 
Nli - au fond, à l'intérieur aussi bien que au sommet, à la base - font bloc contre l'adjonction d'une apposition adjectivale, alors que des formations en dans / sur + le Nli l'autorisent, semble-t-il, un peu mieux [40b], et parfois aussi bien que les emplois non prépositionnels des Nli [41b] :

[40] a. M. Lebigre tenait un fort bel établissement, d'un luxe tout moderne. *Au fond, très sombre, le trou béant de la cave s'ouvrait sous l'escalier tournant, à draperie rouge, qui menait au billard du premier étage.

b. M. Lebigre tenait un fort bel établissement, d'un luxe tout moderne. ?Dans le fond, très sombre, le trou béant de la cave s'ouvrait sous l'escalier tournant, à draperie rouge, qui menait au billard du premier étage.

c. M. Lebigre tenait un fort bel établissement, d'un luxe tout moderne. Le fond, très sombre, était encombré de tables et de chaises.

[41] a. une escarpolette de chocolat [...]*Au sommet, un peu arrondi, étaient posés deux boutons de rose naturels.

b. une escarpolette de chocolat [...] Sur le sommet, un peu arrondi, étaient posés deux boutons de rose naturels.

c. une escarpolette de chocolat $[\ldots]$ Le sommet, un peu arrondi, se terminait par deux boutons de rose naturels.

L'équivalence sémantique, déjà difficile à soutenir entre sur le dessus (de) et le dessus (de), ne peut plus du tout l'être entre au fond (de) et le fond (de), au sommet (de) et le sommet (de).

Lorsque fond, sommet, base, sont utilisés comme de purs Nli, la dimension de «partie intégrante » évoquée par Adler s'efface au profit d'une dimension strictement localisante, comparable à des prépositions/adverbes ou locutions prépositionnelles/adverbiales comme devant, derrière, en haut (de), en bas (de)... Cette valeur strictement localisante exclut l'adjonction d'une apposition et peut rendre difficiles les pronominalisations (du complément - par exemple au moyen du possessif *à son fond, - comme du Nli - p.ex. à celui de $X$ ), qui, elles, ont tendance à activer la dimension partitive du référent. $C f$., à ce sujet, l'exemple [35] ci-dessus et l'exemple [24], répété ici en [42] :

[42] [...] en rentrant les bannes d'osier, la Comtesse vit que le nuage s'élevait et s'étalait ; il s'assombrissait, son fond était très noir mais au sommet il semblait presque vert. (Chandernagor, L'Enfant des lumières, Frantext)

qui oppose la partie du nuage son fond, pour un temps topique du discours, à l'élément localisant au sommet, qui redonne au nuage sa place de topique.

La frontière entre $\mathrm{AA}$ et anaphore à argument nul se révèle finalement difficile à tracer et on observe une forme de continuum ( $c f$. schéma 
1) entre ces anaphores par ellipse, dont les parangons seront les éléments prépositionnels ${ }^{34}$ devant, dessus, et l'AA, illustrée par les emplois non prépositionnels de le fond et les emplois, prépositionnels ou non, des noms de localisés fonctionnels (sous l'escalier tournant). Entre les deux se situent les emplois prépositionnels des Nli, d'abord les formations en $\grave{a}+l e \mathrm{Nli}$ (au fond, à l'intérieur, au sommet), plus figées et plus proches des éléments prépositionnels-adverbiaux, ensuite les autres emplois prépositionnels des Nli (dans le fond, sur le sommet) voire de méronymes ${ }^{35}$ (au second étage, au rez-de-chaussée, sur la plate-forme supérieure), éventuellement combinés avec un adjectif localisant, le numéral ordinal second dans au second étage, l'« adjectif de localisation interne » supérieure (type d'adjectif qui précise une localisation sur la partie, $c f$. Borillo, 1988 et 1998) dans sur la plateforme supérieure. Plus on s'éloigne du pôle associatif, moins l'effet aliénant du défini est sensible, la perte des propriétés anaphoriques du défini s'accentuant en fonction du degré de figement des formations, parallèlement à la perte du statut de syntagme nominal de $l e+$ Nli (laquelle est soulignée par l'impossibilité d'ajouter un syntagme apposé).

Schéma 1 : Continuum anaphorique

\begin{tabular}{|c|c|c|c|}
\hline $\begin{array}{c}\text { Anaphore à } \\
\text { argument nul }\end{array}$ & & & $\begin{array}{c}\text { Anaphore } \\
\text { associative }\end{array}$ \\
\hline $\begin{array}{l}\text { dessus } \\
\text { devant }\end{array}$ & $\begin{array}{c}\text { au fond } \\
\text { au sommet }\end{array}$ & $\begin{array}{c}\text { dans le fond } \\
\text { au second étage }\end{array}$ & $\begin{array}{l}\text { le fond } \\
\text { le sommet }\end{array}$ \\
\hline $\begin{array}{l}\text { Ex. extrait du } \\
\text { Grand Robert de } \\
\text { la langue française } \\
\text { (La Bruyère, Les } \\
\text { Caractères) : Lui- } \\
\text { même écrit une } \\
\text { longue lettre et } \\
\text { met de la poudre } \\
\text { dessus. } \\
\text { Ex. TLFi (Péladan, } \\
\text { Le Vice suprême) : } \\
\text { Six zéros, c'est } \\
\text { néant, mettez un } \\
\text { devant, vous avez } \\
\text { un million. }\end{array}$ & $\begin{array}{l}\text { Ex. [4] : Au fond, } \\
\text { le trou béant de } \\
\text { la cave s'ouvrait } \\
\text { sous l'escalier } \\
\text { tournant }[. . .] \\
\text { Ex. [24] : [...] } \\
\text { mais au sommet } \\
\text { il semblait } \\
\text { presque vert. }\end{array}$ & $\begin{array}{l}\text { Ex. Frantext } \\
\text { (Echenoz, Lac) : } \\
\text { [...] outre un } \\
\text { train de pneus } \\
\text { neige, une } \\
\text { galerie amovible } \\
\text { et deux bidons } \\
\text { de lubrifiant, } \\
\text { des cartons de } \\
\text { vieux livres et } \\
\text { de vieux habits } \\
\text { s'entassaient dans } \\
\text { le fond. } \\
\text { Ex [7] : puis se } \\
\text { tenait au second } \\
\text { étage un donjon } \\
\text { en gâteau de } \\
\text { Savoie [] }\end{array}$ & $\begin{array}{l}\text { Ex. [28] : Le fond } \\
\text { est jaune clair, } \\
\text { bouton d'or. } \\
\text { Ex. Frantext } \\
\text { (Carrère, La Classe } \\
\text { de neige) : Sur la } \\
\text { pente démesurée } \\
\text { de cette dune } \\
\text { descendait une } \\
\text { boule noire. Ce } \\
\text { n'était qu'une petite } \\
\text { tache au début, } \\
\text { quand elle quittait } \\
\text { le sommet, mais } \\
\text { elle devenait de } \\
\text { plus en plus grosse } \\
\text { en dévalant [] }\end{array}$ \\
\hline
\end{tabular}

Ces différentes anaphores constituent autant d'organisateurs textuels de l'espace possibles : des plus figés - anaphores à argument nul : devant, derrière, à droite... - aux plus libres - AA prépositionnelles avec nom 
de localisé fonctionnel : sous l'escalier tournant en [4] - en passant par ces anaphores prépositionnelles 'intermédiaires', construites avec un nom relationnel (Nli ou méronyme), les plus figées (au fond, à l'intérieur, au sommet) se rapprochant davantage du pôle 'anaphore à argument nul'.

\section{Conclusion}

Dans les anaphores prépositionnelles intermédiaires, entre AA et anaphore à argument nul, le défini n'a pas l'effet focalisant qu'il a dans les AA : il ne s'agit plus de faire un gros plan sur un objet ou une partie, mais d'introduire un nouveau cadre. En position initiale détachée (voir notamment Charolles et Vigier, 2005), cette propriété confère probablement à ces anaphores un fort pouvoir cadratif, dont la portée risque toutefois d'être souvent limitée, à cause de la brièveté des descriptions (c'est le cas des exemples qu'on a pu relever dans Frantext) ou de la fréquence des changements de cadre dans des descriptions aussi dynamiques que celles de [4] et [7], cette dernière étant un modèle de description ascendante. Une étude plus précise du fonctionnement de ces anaphores 'de cadre' dans les descriptions spatiales et de leur influence sur la 'dynamicité' descriptive reste à faire.

\section{NOTES}

1. L'Elaboration ou é-élaboration et l'Elaboration d'entité ou i-élaboration sont des relations de spécification qui manifestent une différence de portée ( $c f$. Kleiber et Vassiliadou, 2007 : 154 et 2009 : 189) : la spécification, l'ajout de détails porte sur la situation entière de la phrase élaborée pour la première et seulement sur (un de) ses actants pour la seconde. Si l'exemple [1] illustre une relation d'Elaboration d'entité, l'exemple suivant, emprunté à Kehler (2002 : 18), illustre une relation d'Elaboration stricte : A young aspiring politician was arrested in Texas today. John Smith, 34, was nabbed in a Houston law firm while attempting to embezzle funds for his campaign. La deuxième phrase, tout en conduisant à la même proposition que la première ('un jeune politicien a été arrêté'), ajoute des détails et des informations qui concernent non seulement le principal protagoniste (son nom et son âge), mais aussi les circonstances de l'événement (le lieu et le motif de l'arrestation).

2. Ou encore comme la description d'œuvre d'art - ou ekphrasis - suivante (il s'agit d'un tableau de Grünewald) :

[...] le Christ se dressait, formidable, sur sa croix [...] L'aisselle éclamée craquait ; les mains grandes ouvertes brandissaient des doigts hagards qui bénissaient quand même, dans un geste confus de prières et de reproches, les pectoraux tremblaient, beurrés par les sueurs; ;e torse était rayé de cercles de douves par la cage divulgée des côtes; les chairs gonflaient, salpêtrées et bleuies, persillées de morsures de puces, mouchetées comme de coups d'aiguilles par les pointes des verges qui, brisées sous la peau, la lardaient encore, çà et là, d'échardes. (Huysmans, Là-Bas, Folio/Gallimard, 1991 : 33). 
3. Au sens de Sanders, Spooren et Noordman (1992a et b). L'opposition entre source sémantique et source pragmatique de la cohérence constitue l'un des quatre primitifs fondant leur classification des relations de cohérence. Comme ils le signalent eux-mêmes (1992a : 8), cette distinction est comparable à celle de van Dijk (1979) - déjà en termes de « sémantique » vs « pragmatique »- ou encore à celle qu'utilisent Halliday et Hasan (1976) ou Martin (1983) entre relations « externes » ou « internes » à la situation de communication. $C f$. aussi, chez Mann et Thompson (1988), la distinction entre relations de contenu et relations de présentation.

4. Une autre preuve, qui n'est pas cette fois apportée par une AA : les anémones éparpillées dans l'herbe.

5. Les AA méronymiques reposent sur une relation partie-tout au sens strict, répondant au cadre générique Un X est une partie d'un Y (cf. Cruse, 1986 et Kleiber, 2001), p.ex. Un tronc est une partie d'un arbre; Un moteur est une partie d'une voiture. Les AA locatives reposent, quant à elles, sur une relation de localisation fonctionnelle. Les entités englobantes sont ici, suivant les termes de Kleiber (2001 : 292), « des lieux prototypiques pour la fonctionnalité inhérente des entités $\mathrm{X}$ [= les entités incluses], c'est-à-dire des lieux qui, par avance ou a priori, sont destinés à accueillir les entités $X$. » Cette relation répond au cadre générique Dans un Y, il y a un X (cf. Kleiber, 2001 : 300), p.ex. Dans un village, il y a une église. Ce cadre s'applique aussi à certaines relations méronymiques (par exemple, moteur / voiture), mais, contrairement à ces dernières, les relations locatives n'autorisent pas le cadre Un X est une partie d'un Y (?Une église est une partie d'un village).

6. Dans chacun de ces exemples, on pourrait insérer le connecteur en effet, proposé par Cornish (2009b), comme indice possible de la relation Assertion-Indice, avant la séquence anaphorique.

7. Détachement qui, avec des parties inaliénables comme le sont les parties du corps, ne peut guère être qu'un détachement visuel. Sur ce point, $c f$. Kleiber (1999 : 88) : « l'aliénation en pensée ou imaginaire exigée par l'anaphore associative est celle de la perception visuelle. Le système visuel permet d'isoler ou de détacher un élément sur une situation ou un objet sans que l'élément ainsi aliéné cesse pour autant de faire partie de la situation ou de l'objet.»

8. Selon Borillo (1988), les noms de localisation interne désignent des zones particulières d'un objet-site et permettent de localiser une entité (la cible) en contact ou à l'intérieur de cet objet. Dans les exemples suivants (empruntés à Borillo, 1988 : 6) : Le plat est sur le bord de la table; Le livre est au fond de la bibliothèque, les Nli bord et fond permettent de localiser les entités-cibles 'plat' et 'livre' de manière plus précise que des syntagmes prépositionnels en sur ou dans (sur la table, dans la bibliothèque), puisqu'ils mentionnent non pas l'ensemble de l'objet-site, mais une zone particulière de celui-ci.

9. Ce dernier Nli, comme me le fait remarquer un relecteur, ne se rattache plus directement à la pièce montée, mais à certaines de ses parties (les deux poteaux ou l'escarpolette).

10. Syntagmes libres, AA, et non locutions, selon Adler (2008) et son critère d'équivalence sémantique.

11. En fait, comme l'a souligné Kleiber (2001 : 309), le possessif est toujours possible lorsqu' on a affaire à des relations méronymiques au sens strict (une voiture / son moteur, un arbre / ses racines), mais pas toujours lorsqu'il s'agit de relations 
locatives (un village / son église, une cuisine / ?son réfrigérateur ; ces deux exemples sont repris à Kleiber, $2001: 309$, note 1 ).

12. Qui s'appuie ici sur des relevés effectués dans Frantext.

13. L'AA que pourtant Aurnague (1996) exclut avec les Nli, en s'appuyant sur l'impossibilité de construire une AA telle que un village ??les sommets (opposée à l'acceptabilité de un village... les toits). Mais ici, le problème ne relève pas du statut de Nli de sommet mais de sa variabilité sémantique (Aurnague met d'ailleurs lui-même en avant le caractère général des Nli pour expliquer l'impossibilité de ce type d'AA) et du « saut » demandé. Cette variabilité est assez largement partagée par les Nli puisque la valeur relationnelle de la plupart d'entre eux s'étend à nombre de noms : tous les noms d'objets bidimensionnels, par exemple, compteront milieu, bout, extrémité parmi leurs Nli (cf. Borillo, 1998 : 68). Cette propriété interdit le saut de village à sommets sans nommer les entités intermédiaires maisons, bâtiments. Mais cette interdiction n'est ni propre à l'AA (un village ??ses sommets, ??les sommets $d u$ village ne sont pas meilleurs) ni propre aux Nli. Des blocages de transitivité comparables sont en effet observés avec les méronymes, comme dans le célèbre exemple de Lyons (1977) : la maison a une porte / la porte a une poignée / *la maison a une poignée ; sur ces questions, voir Cruse (1979) et (1986), Schnedecker (1997) et Salles (2000).

14. Préposition qui, selon Vandeloise (1988), sélectionne dans ses emplois spatiaux des entités remplissant une « fonction de localisation », c'est-à-dire des entités-sites fixes et dont « la position est connue dans la connaissance partagée » (Aurnague, 1996 : 162). Cette fonction de localisation permet d'opposer objets et lieux «sur la base des critères d'immobilité et de spécification de la position » (Aurnague, 1996 : 164). La différence entre angle, coin d'un côté et arête de l'autre est, selon Aurnague (1996 : 181), liée au fait que arête, contrairement à angle et coin, ne peut pas localiser une cible qui ne serait pas en contact avec le site et ne peut ainsi « faire référence à une portion d'espace adjacente ».

15. En fait, Aurnague (1996 : 178) estime cette pronominalisation par celui à l'intérieur d'un groupe prépositionnel en sur « délicate » pour un méronyme et « clairement pas possible » pour un Nli.

16. Ce test de la pronominalisation est d'ailleurs utilisé par Borillo (1992) à la fois pour distinguer Nli et méronymes, et locutions prépositionnelles et séquences libres.

17. Comme le précise Aurnague (1996), haut acceptera ces emplois lorsqu'il abandonnera sa fonction première de localisation interne pour jouer le rôle d'un composant, autrement dit lorsqu'il ne sera plus Nli mais méronyme. C'est, par exemple, le cas lorsqu'il désigne l'une des parties d'un maillot de bain.

18. Spécialisation qui n'empêche toutefois pas des emplois plus originaux comme la lisière d'un rectangle de lumière et la lisière du danger des exemples [20] et [21] donnés plus loin.

19. Cf. encore Husserl (1962: 12) : «Pouvoir nous représenter 'séparément' ou en 'elle-même' la tête d'un cheval, cela signifie que nous pouvons la retenir dans notre imagination, pendant que nous laissons se modifier librement et disparaître les autres parties du cheval et tout l'environnement intuitif. »

20. Sur la question de l'AA et des noms de propriétés, voir Kleiber (1999 et 2001).

21 . Et, parmi ces 46 occurrences, plus de la moitié (28 exactement) sont précédées de la préposition à ; 4 seulement ne sont pas régimes d'une préposition. 
22. Selon Huyghe (2005: 193), les Nli refuseraient aussi en. Huyghe oppose ??Le fond en est sale à La poignée en est cassée. Jugement d'acceptabilité encore une fois discutable : Le fond / Le haut / Le bas en est sale me semblent tous possibles. Et les exemples suivants ne paraissent pas exceptionnels : Je lui fis un matelas en fibres de coco, des coffrets de cyprès mêlé du vétiver qui parfume à jamais, et j'en couvrais le fond avec de l'acajou [...] (Chamoiseau, Texaco, Frantext) ; [ ] une demi-douzaine de tasses à café, leur petite cuillère, parierait-il, tenant debout toute seule dans le mélange de marc et de sucre fondu qui en constitue le fond [...] (Benoziglio, La voix des mauvais jours et des chagrins rentrés, Frantext).

23. Un relecteur trouve cependant son avant douteux et suggère une recherche dans ma sélection de romans. On ne trouve qu'une occurrence de son avant (précédé d'une préposition non spatiale) : cette jonque irréelle, à la coque brune, avec son avant ouvert en ailes qui laisse voir le guindeau et l'ancre lestée (Dormann, La Petite main, Frantext). Mais, si l'on relève de nombreuses occurrences de l'avant précédé d'une préposition spatiale (essentiellement à et vers), on ne relève que deux emplois non prépositionnels de ce Nli au défini : [...] le camion dont parlait Dimeglio. L'avant était tourné vers l'entrée du hangar [ ] (Jonquet, Les Orpailleurs, Frantext); Après l'avoir terminée, j'ai essayé de tourner la guêpière de façon que l'avant et l'arrière retrouvent leur juste place en vain! (Dorin, Les Vendanges tardives, Frantext).

24. $C f$. aussi les remarques d'Aurnague (1996) déjà évoquées (note 17) à propos de l'emploi méronymique de haut, par exemple dans un haut de maillot de bain.

25. A ce propos, on notera que les trois emplois anaphoriques (i.e. sans complément du nom en de) du Nli lisière, que l'anaphore soit définie ou possessive, sont bien ou prépositionnels (à sa lisière, à la lisière en [20] et [21]) ou complément d'un verbe de mouvement (longeait la lisière en [19]).

26. La préposition la plus fréquente est la préposition à. Dans la même sélection, on peut relever 845 au fond. Si l'on examine les 300 premières occurrences, on trouve 19 occurrences associatives (textuelles ou quelquefois situationnelles, comme dans Assieds-toi au fond), ce qui est finalement assez peu et, en tout cas, sans commune mesure avec le nombre de au fond de et de au fond, locution adverbiale abstraite que les dictionnaires glosent généralement par 'en réalité'.

27. La même sélection propose 89 résultats pour au sommet.

28. Situationnel, par exemple, dans : Vous êtes malade, Monsieur ? [...] - Ça va, merci. - En cas de besoin, tapez sur le mur du fond, je suis infirmière. (Schreiber, Un silence d'environ une demi-heure, Frantext)

29. Je désigne ainsi les entités incluses ('église' par rapport à 'village', 'réfrigérateur' par rapport à 'cuisine') des relations locatives de Kleiber (2001).

30. Encore une fois au sens large du terme, incluant les localisés fonctionnels.

31. Toutes les prépositions spatiales n'excluent toutefois pas la valeur méronymique. Dans un cas lexicalement plus tranché que nos exemples, Aurnague (1996: 168) distingue la préposition sur, + méronyme [a], et la préposition $\grave{a},+\mathrm{Nli}[\mathrm{b}]$ : [a] L'étiquette est sur le pied de la table; [b] L'étiquette est au pied de la table.

32. Bien qu'elle ne soit pas impossible, comme le souligne un relecteur qui en a relevé de nombreux exemples sur Internet.

33. Et « l'effacement des propriétés substantives du nom» (Huyghe, 2005 : 202) est souligné par l'impossibilité de le pronominaliser (au fond de l'établissement et *à celui du magasin ; à l'intérieur de la chambre et *à celui du bureau), impossibilité ou difficulté déjà constatée avec l'exemple [15]. 
34. On peut considérer, à l'instar de Borillo (1992 : 185), qu'il s'agit toujours de prépositions, qu'ils soient ou non suivis d'un complément, étant donné que même « privée de son complément, la PREPLI [= préposition ou locution prépositionnelle de localisation interne] reste toujours un élément relationnel qui doit se rapporter à un SN représentant l'objet-site ». L'analyse en termes d'anaphore à argument nul suggère aussi un tel statut.

35. Mais pas de noms de localisés fonctionnels : non relationnels ( $c f$. Kleiber, 2001), ils excluent l'anaphore à argument nul et n'autorisent qu'une interprétation purement associative.

\section{RÉFÉRENCES}

ADAM J.-M., 1993, La description, Paris, Paris, PUF.

ADAm J.-M., 2005, La linguistique textuelle. Introduction à l'analyse textuelle des discours, Paris, Armand Colin.

AdLer S., 2006, «L'emploi absolu d'expressions de lieu de type [PREP LE NOM $D E]$ : ellipse ou anaphore associative ? », Langages, 169, p. 67-81

AdLer S., 2008, «Evénementialité et partitivité dans les séquences [PREP LE NOM $D E$ ] temporelles », in Kleiber, G, Schnedecker, C. et Theissen, A., La relation partie-tout, Louvain, Peeters, p. 259-272.

Aurnague M., 1989, "Catégorisation des objets dans le langage : les noms de localisation interne », Cahiers de grammaire, 14, p. 1-21.

Aurnague M., 1996, « Les noms de localisation interne : tentative de caractérisation sémantique à partir de données du basque et du français », Cahiers de lexicologie, 69, 2, p. 159-192.

Borillo A., 1988, « Le lexique de l'espace : les noms et les adjectifs de localisation interne », Cahiers de grammaire, 13, p. 1-22.

Borillo A., 1992, «Le lexique de l'espace : prépositions et locutions prépositionnelles de lieu en français », in TAsmowski, L. et Zribi-Hertz, A., De la musique à la linguistique. Hommages à Nicolas Ruwet, Gand, Communication et Cognition, p. 176-190.

Borillo A., 1998, L'espace et son expression en français, Gap, Paris, Ophrys.

Borillo A., 1999, "Partition et localisation spatiale : les noms de localisation interne », Langages, 136, p. 53-75.

Charolles M. et Vigier D., 2005, «Les adverbiaux en position préverbale : portée cadrative et organisation des discours », Langue française, 148, p. 9-30.

CoRNISH F. 2009a, « Inter-sentential anaphora and coherence relations in discourse: a perfect match », Language Sciences, 31, p. 572-592.

Connish F., 2009b, « Le rôle des anaphores dans la mise en place des relations de cohérence dans le discours : l'hypothèse de J.R. Hobbs », Journal of French Language Studies, 19, 2, p. 159-181.

CRuse D. A., 1979, « On the transitivity of the part-whole relation », Journal of Linguistics, 15, 1, p. 29-38.

CRuse D.A., 1986, Lexical semantics, Cambridge, Cambridge University Press.

Eco U., 2009, Vertige de la liste, traduction française M. Bouzaher, Paris, Flammarion. 
Fabricius-Hansen C. et Behrens B., 2001, « Elaboration and related discourse relations viewed from an interlingual perspective », Sprik reports, 13, p. 1-34. http://www.hf.uio.no/german/sprik.

Fontanier, P., 1977, Les figures du discours (1821-1830), Paris, Flammarion.

Halliday M.A.K. et Hasan R., 1976, Cohesion in English, London, Longman.

Husserl E., 1962, Recherches logiques, Tome II, deuxième partie, Paris, PUF.

Huyghe R., 2005, «Zones et parties : l'hétérogénéité des « noms de localisation interne » », Le français moderne, 73, 2, p. 184-211.

KeHLER A., 2002, Coherence, Reference, and the Theory of Grammar, Stanford, CSLI Publications.

KLEIBER G., 1999, « Anaphore associative et relation partie-tout : condition d'aliénation et principe de congruence ontologique », Langue française, 122, p. 70-100.

KLEIBER G., 2001, L'anaphore associative, Paris, PUF.

Kleiber G. et Vassiliadou H., 2007, « Sur les approches intuitives de la relation d'Elaboration », Scolia, 22, p. 147-161.

KLeiber G. et Vassiliadou H., 2009, « Sur la relation d'Elaboration : des approches intuitives aux approches formelles », Journal of French Language Studies, 19, 2, p. 183-205.

Lyons J., 1977, Semantics I, Cambridge, Cambridge University Press.

Mann W.C. et Thompson S.A., 1988, « Rhetorical Structure Theory: Toward a functional theory of text organization », Text, 8, 3, p. 243-281.

Martin J.R., 1983, « Conjonction: The logic of English text », in PetöFi J.S. et Sözer E., Micro and macroconnexity of texts, Hamburg, Buske, p. 1-72.

Prévot L., Vieu L. et Asher N., 2009, « Une formalisation plus précise pour une annotation moins confuse : la relation d'Elaboration d'entité », Journal of French Language Studies, 19, 2, p. 207-228.

Salles M., 2000, «La transitivité de la relation lexicale partie-tout », Le français moderne, 68, 2, p. 171-190.

Sanders T., Spooren W. et Noordman L., 1992a, " Toward a taxonomy of coherence relations », Discourse Processes, 15, p. 1-35.

Sanders T., Spooren W. et Noordman L., 1992b, « Coherence relations in a cognitive theory of discourse representation », Cognitive linguistics, 4, 2, p. 93-133.

SCHNEDECKER C., 1997, «A quelles conditions l'anaphore associative peut-elle être transitive ? Quelques suggestions... », in De Mulder W., TAsmowski L. et Vetters C., Relations anaphoriques et (in)cohérence, Amsterdam, Rodopi, p. $99-116$.

THEISSEN A., 2001a, « La concurrence entre un SN défini fidèle et un SN défini totalement fidèle ou Comment expliquer le maintien ou non de l'adjectif dans un SN défini anaphorique basique ? », in De Mulder W., Vet C. et Vetters C., Anaphores pronominales et nominales. Etudes pragma-sémantiques, Amsterdam, Rodopi, p. 43-67.

Theissen A., 2001b, «Quelques observations sur l'apparition d'un adjectif dans un SN défini de reprise », in Buridant C., Kleiber G. et Pellat J.-C., Par monts et par vaux. Itinéraires linguistiques et grammaticaux (Mélanges offerts à Martin Riegel), Louvain-Paris, Peeters, p. 407-416.

VAndeloise C., 1988, «Les usages statiques de la préposition à », Cahiers de lexicologie, 53, p. 119-148.

VAN DiJK T. A., 1979, « Pragmatic connectives », Journal of Pragmatics, 3, p. 447-456. 